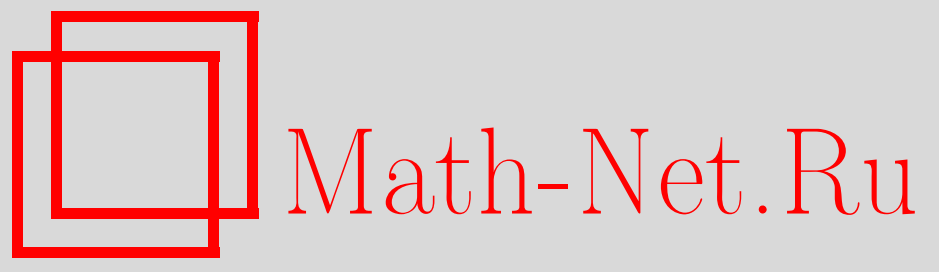

М. С. Ермаков, Метод существенной выборки для моделирования вероятностей умеренных и больших уклонений оценок и критериев, Теория вероятн. и ее примен., 2006, том 51, выпуск 2, 319-332

DOI: https://doi.org/10.4213/tvp56

Использование Общероссийского математического портала Math-Net.Ru подразумевает, что вы прочитали и согласны с пользовательским соглашением

http://www.mathnet.ru/rus/agreement

Параметры загрузки:

IP : 18.208 .226 .222

26 апреля 2023 г., 02:23:22

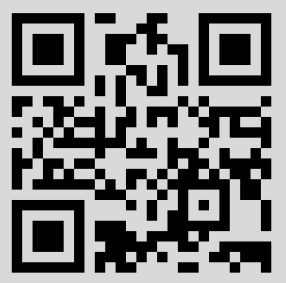




\section{МЕТОД СУЩЕСТВЕННОЙ ВЫБОРКИ ДЛЯ МОДЕЛИРОВАНИЯ ВЕРОЯТНОСТЕЙ УМЕРЕННЫХ И БОЛЬШИХ УКЛОНЕНИЙ ОЦЕНОК И КРИТЕРИЕВ}

В работе изучается эффективность метода существенной выборки для вычисления вероятностей больших и умеренных уклонений статистических оценок и тестовых статистик. В отличие от прямого моделирования объем моделирования рассмотренных эффективных процедур сушественной выборки не имеет экспоненциального роста. Результаты применяются к моделированию вероятностей умеренных уклонений критериев типа омега-квадрат.

Ключевые слова и фразы: метод существенной выборки, вероятности больших уклонений, вероятности умеренных уклонений, эмпирическая мера.

1. Введение. При непосредственном моделировании редких событий вероятность появления событий мала. Поэтому для решения этой задачи довольно часто применяются специальные методы моделирования. Наиболее распространенным из них является метод сушественной выборки (см. [4], [16], [10]). Для вычисления вероятности редкого события случайные величины в этом методе моделируются в соответствии с некоторым другим вероятностным распределением. После этого наблюдениям приписываются веса обусловленные переходом к новой вероятностной мере моделирования.

Задача выбора оптимальной меры в процедуре существенной выборки была исследована для широкого круга приложений (см. [15], [4], [5], [14], [6], [13], [12], [2], [16]). Оптимальные меры моделирования находились на основе методов теории вероятностей больших уклонений сумм независимых случайных величин. Статистические процедуры обычно имеют только приближенно линейный или даже нелинейный характер и не могут изучаться на основе такой техники без привлечения какоголибо дополнительного аппарата. Как следствие, подобные результаты в статистике получены только для специальных моделей (см. [15], [14], [2]).

Как известно, статистические оценки и тестовые статистики обычно могут быть представлены в виде функционалов от эмпирической

* Институт проблем машиноведения РАН, Большой просп. В.О., 61, 199178 С.-Петербург, Россия; e-mail: ermakov@random.ipme.ru 
меры. Поэтому представляется естественным проанализировать эффективность метода существенной выборки на основе теорем о вероятностях больших и умеренных уклонений эмпирических мер (см. [9], [3], [8]). Этому вопросу и посвящена настоящая работа. Результаты для процедуры эффективного моделирования вероятностей больших уклонений получены в терминах информационных чисел Кульбака-Лейблера и допускают наглядную интерпретацию: вероятностные меры для моделирования методом сушественной выборки являются решением экстремальной задачи минимизации информационных чисел Кульбака-Лейблера на соответствующих множествах больших уклонений вероятностных мер. Хотя непосредственное вычисление информационных чисел КульбакаЛейблера и соответствующих вероятностных мер для эффективного моделирования представляет существенную трудность, эти результаты могут быть использованы для получения приближенных решений.

Трудности, возникающие при эффективном моделировании вероятностей больших уклонений, стали одной из причин исследования аналогичной задачи в зоне умеренных уклонений. Зоны умеренных уклонений статистик обычно могут быть аппроксимированы полупространствами или выпуклыми множествами в множестве всех вероятностных мер. Как следствие, в приложениях проверка условий теорем об эффективном моделировании вероятностей умеренных уклонений не представляет таких серьезных трудностей, как в случае больших уклонений. Естественно, задача моделирования умеренных уклонений имеет также и независимый интерес для приложений. Результаты для этой задачи выражены в терминах расстояния Хеллингера и функционала, допускающего интерпретацию в статистических задачах как функционал информационного количества Фишера.

Отметим, что интересные результаты по эффективному моделированию на основе метода сушественной выборки были получены в [11] и [10]. В этих работах задача моделирования рассматривалась в зоне $O\left(n^{-1 / 2}\right)$-уклонений.

2. Метод существенный выборки для вероятностей больших уклонений. Пусть $X_{1}, \ldots, X_{n}$ - независимые одинаково распределенные случайные величины, имеющие вероятностную меру $P$, заданную на сигма-алгебре борелевских множеств $\Im$ в топологическом пространстве Хаусдорфа $S$. Обозначим $\widehat{P}_{n}$ эмпирическую меру $X_{1}, \ldots, X_{n}$. Определим множество $\Lambda$ всех вероятностных мер на $(S, \Im)$.

Для любых $P, Q \in \Lambda$ информационное число Кульбака-Лейблера задается равенством

$$
K(Q, P)=\int_{S} q \ln q d P, \quad q=\frac{d Q}{d P}
$$

если $Q$ абсолютно непрерывна относительно $P$, и $K(Q, P)=\infty$ в про- 
тивном случае. Для любых $P \in \Lambda$ и $\Omega \subset \Lambda$ обозначим $K(\Omega, P)=$ $\inf \{K(Q, P): Q \in \Omega\}$.

Определим $\tau$-топологию слабой сходимости в $\Lambda$ такую, что последовательность вероятностных мер $Q_{n} \in \Lambda$ сходится к вероятностной мере $Q \in \Lambda$ в $\tau$-топологии, если и только если $\lim _{n \rightarrow \infty} \int_{S} f d Q_{n}=\int_{S} f d Q$ для каждой ограниченной измеримой функции $f: S \rightarrow \mathbf{R}^{1}$. В дальнейшем все топологические свойства в $\Lambda$ (сходимость, замкнутость, компактность и т.д.) будут рассматриваться относительно $\tau$-топологии. Замкнутые и открытые множества $\Omega \subset \Lambda$ в $\tau$-топологии будут обозначаться $\operatorname{cl}(\Omega)$ и $\operatorname{int}(\Omega)$ соответственно.

Пусть дан функционал $T: \Lambda \rightarrow \mathbf{R}^{1}$. Для каждого $b>0$ определим множество $\Omega(b)=\{Q: T(Q)>b, Q \in \Lambda\}$.

Дальнейшие рассуждения будут базироваться на следующих теоремax (см. лемму 2.3, теоремы 3.1 и 3.2 ) в [9]).

Теорема 2.1. Для любой вероятностной мерь $P \in \Lambda$ и множества $\Omega \subset \Lambda$ справедлив приниип больших уклонений

$$
\begin{gathered}
\liminf _{n \rightarrow \infty} n^{-1} \ln P\left(\widehat{P}_{n} \in \Omega\right) \geqslant-\dot{K}(\operatorname{int}(\Omega), P), \\
\limsup _{n \rightarrow \infty} n^{-1} \ln P\left(\widehat{P}_{n} \in \Omega\right) \leqslant-K(\operatorname{cl}(\Omega), P) .
\end{gathered}
$$

Существует вероятностная мера $Q \in \operatorname{cl}(\Omega)$ такая, что $Q$ абсолютно непрерывна относительно $P$ и $K(Q, P)=K(\operatorname{cl}(\Omega), P)$.

Теорема 2.2. Пусть функиионал $T: \Lambda \rightarrow \mathbf{R}^{1}$ непрерьвен в $\tau$-топологии, и пусть функиия $t \rightarrow K(\Omega(t), P)$ непрерывна справа в точке $t=b$. Тогдa

$$
\lim _{n \rightarrow \infty} n^{-1} \ln P\left(T\left(\widehat{P}_{n}\right)>b\right)=-K(\Omega(b), P) .
$$

Существует вероятностная мера $Q_{b} \in \operatorname{cl}(\Omega(b))$ такая, что $K(\Omega(b), P)=K\left(Q_{b}, P\right)$.

Мы рассматриваем следующий класс процедур существенной выборки. Возьмем вероятностные меры $Q_{1}, \ldots, Q_{l} \in \Lambda$, абсолютно непрерывные относительно вероятностной меры $P$, и неотрицательные целые числа $p_{1}, \ldots, p_{l}$ такие, что $p_{1}+\cdots+p_{l}=1$. Осуществим моделирование $t$ независимых выборок $Y_{1}^{(i)}, \ldots, Y_{n}^{(i)}, 1 \leqslant i \leqslant t$, независимых случайных величин, имеющих вероятностную меру $Q=p_{1} Q_{1}+\cdots+p_{l} Q_{l}$. Отметим, что вероятностная мера $Q$ может моделироваться на основе моделирования вероятностной меры $Q_{\nu}$, где $\nu$ - случайный индекс, $P(\nu=j)=p_{j}, 1 \leqslant j \leqslant l$.

Обозначим $\widehat{Q}_{n}^{(i)}$ эмпирическую меру $Y_{1}^{(i)}, \ldots, Y_{n}^{(i)}, 1 \leqslant i \leqslant t$.

Оценка метода существенной выборки для $P\left(T\left(\widehat{P}_{n}\right)>b\right)$ равна

$$
\widehat{V}_{n t}=t^{-1} \sum_{i=1}^{t} \chi\left(T\left(\widehat{Q}_{n}^{(i)}\right)>b\right) w_{n i}^{-1}
$$


где

$$
w_{n i}=\sum_{j=1}^{l} p_{j} u_{n i j}, \quad u_{n i j}=\prod_{s=1}^{n} q_{j}\left(Y_{s}^{(i)}\right)
$$

и $q_{j}=d Q_{j} / d P, 1 \leqslant j \leqslant l$.

Задание вероятностной меры $Q$ как смеси вероятностных мер будет необходимо в дальнейшей постановке задачи.

Непосредственными вычислениями получаем

$$
E_{Q} \widehat{V}_{n t}=E_{Q} \widehat{V}_{n 1}=P\left(T\left(\widehat{P}_{n}\right)>b\right)
$$

И

$$
\begin{aligned}
D_{Q} \widehat{V}_{n t} & =t^{-1}\left(E_{Q} \widehat{U}_{n}-\left(E_{Q} \widehat{V}_{n 1}\right)^{2}\right) \\
& =t^{-1}\left(E_{P}\left[w_{n 1}^{-1} \chi\left(T\left(\widehat{Q}_{n}^{(1)}\right)>b\right)\right]-\left(P\left(T\left(\widehat{P}_{n}\right)>b\right)\right)^{2}\right),
\end{aligned}
$$

где $\widehat{U}_{n}=\chi\left(T\left(\widehat{Q}_{n}^{(1)}\right)>b\right) w_{n 1}^{-2}$.

Из (2.5) следует, что процедура метода существенной выборки является несмещенной и дает оценку искомой вероятности.

По теореме 2.2 и (2.5) получаем

$$
E_{Q} \widehat{V}_{n 1}=\exp \{-n K(\Omega(b), P)(1+o(1))\}
$$

при $n \rightarrow \infty$.

Из (2.1), (2.5)-(2.7) вытекает следующая лемма.

Лемма 2.1. Пусть функиионал $T: \Lambda \rightarrow \mathbf{R}^{1}$ непрерьвен в $\tau$-топологии и $K\left(\operatorname{cl}\left(\Omega_{b}\right), P\right)=K\left(\operatorname{int}\left(\Omega_{b}\right), P\right)$. Тогда для любых $Q_{1}, \ldots, Q_{l} \in \Lambda$ и неотричательных чисел $p_{1}, \ldots, p_{l}$ таких, что $p_{1}+\cdots+p_{l}=1$, выполнено неравенство

$$
\liminf _{n \rightarrow \infty} n^{-1} \ln E_{Q} \widehat{U}_{n} \geqslant-2 K(\Omega(b), P) .
$$

Утверждение леммы 2.1 позволяет ввести естественное определение асимптотической эффективности процедур существенной выборки. Скажем, что процедура существенной выборки $\widehat{V}_{n t}$ является асимптотически эффективной, если

$$
\lim _{n \rightarrow \infty} n^{-1} \ln E_{Q} \widehat{U}_{n}=-2 K(\Omega(b), P) .
$$

Аналогичное понятие асимптотической эффективности в несколько других терминах использовалось в [4] и [14]. Грубо говоря, для асимптотически эффективной процедуры существенной выборкй объем вычислений растет медленнее, чем экспоненциально.

Для множества $\Omega \subset \Lambda$ оценки $P\left(\widehat{P}_{n} \in \Omega\right)$ определяются аналогично:

$$
\widehat{V}_{n t}=t^{-1} \sum_{i=1}^{t} \chi\left(\widehat{Q}_{n}^{(i)} \in \Omega\right) w_{n i}^{-1} .
$$

Для этой постановки также справедлив аналог леммы 2.1 в следующих обозначениях: $\widehat{U}_{n}=w_{n 1}^{-2} \chi\left(\widehat{Q}_{n}^{(1)} \in \Omega\right)$ и $\Omega(b)=\Omega$. Следовательно, для этой 
задачи может быть введено аналогичное определение асимптотической эффективности. Естественно, процедура существенной выборки для вычисления вероятностей больших уклонений $P\left(T\left(\widehat{P}_{n}\right)>b\right)$ может рассматриваться как частный случай (2.10). Достаточно положить $\Omega=\Omega(b)$.

Предположим, что вероятностная мера $R \in \Lambda$ абсолютно непрерывна относительно вероятностной меры $P \in \Lambda$, и обозначим $r=$ $d R / d P$. Положим $\Gamma_{R}=\left\{Q: \int_{S} \ln r d Q>K(\Omega, P), Q \in \Lambda\right\}$.

Теорема 2.3. Пусть $\Omega \subset \Lambda u P \in \Lambda$. Пусть $K(\operatorname{cl}(\Omega), P)=$ $K(\operatorname{int}(\Omega), P)$. Предположим, что существует только конечное число вероятностных мер $R_{1}, \ldots, R_{m}$ таких, что $R_{i} \in \operatorname{cl}(\Omega) u K\left(R_{i}, P\right)=$ $K(\Omega, P)$ для всех $1 \leqslant i \leqslant m$. Обозначим $r_{i}=d R_{i} / d P$ и предположим, что $C>r_{i}(x)>c>0$ для всех $x \in S, 1 \leqslant i \leqslant m$. Предположим также, что $\Omega \subset \bigcup_{i=1}^{m} \Gamma_{R_{i}}$. Тогда процедура существенной вььборки (2.4) является асимптотически эффективной для данных вероятностных мер $Q_{1}, \ldots, Q_{l}$, если и только если множество вероятностных мер $Q_{1}, \ldots, Q_{l}$ содержит множество $R_{1}, \ldots, R_{m} u p_{j} \neq 0$ для всех $1 \leqslant j \leqslant l$ таких, что $Q_{j}=R_{i_{j}}$ при некотором $i_{j}, 1 \leqslant i_{j} \leqslant m$.

Предположим, что Функиионал $T: \Lambda \rightarrow \mathbf{R}^{1}$ непрерывен в $\tau$-топологии. Тогда $K(\operatorname{cl}(\Omega(b)), P)=K(\operatorname{int}(\Omega(b)), P)$, если отображение $t \rightarrow K(\Omega(t), P)$ непрерьвно справа в точке $t=b$. Следовательно, если множество $\Omega=\Omega($ b) удовлетворяет всем остальным условиям теоремь, то ее утверждение для него выполнено.

Доказательство теоремы 2.3 опускается. Аналогичные рассуждения проведены в доказательстве теоремы 3.2 (п. 4) для случая вероятностей умеренных уклонений. Доказательство теорем использует как технику анализа вероятностей больших уклонений вероятностных мер (см. [9], [7], [8]), так и методы, развитые для доказательства асимптотической эффективности процедуры существенной выборки (см. [14], [13] и ссылки в этих работах).

\section{3. Метод сушественной выборки для вероятностей умерен-} ных уклонений. Будем предполагать, что функционал $T: \Lambda \rightarrow \mathbf{R}^{1}$ непрерывен в $\tau$-топологии и вероятностная мера $P$ является предельной точкой последовательности множеств $\Omega_{n} \subset \Lambda$, причем $P \notin \Omega_{n}$. Пусть $T(P)=0$, и пусть $b_{n}>0, b_{n} \rightarrow 0, n b_{n}^{2} \rightarrow \infty$ при $n \rightarrow \infty$. В этом пункте мы найдем эффективные процедуры для оценивания вероятностей умеренных уклонений $P\left(T\left(\widehat{P}_{n}\right)>b_{n}\right)$ и $P\left(\widehat{P}_{n} \in \Omega_{n}\right)$.

Для любых $P, Q \in \Lambda$ определим расстояние Хеллингера

$$
\rho(Q, P)=\left(\int_{S}\left(\left(\frac{d Q}{d R}\right)^{1 / 2}-\left(\frac{d P}{d R}\right)^{1 / 2}\right)^{2} d R\right)^{1 / 2}, \quad R=\frac{1}{2}(P+Q) .
$$

Для любого $\Omega \subset \Lambda$ обозначим $\rho(\Omega, P)=\inf \{\rho(Q, P): Q \in \Omega\}$. 
Определим пространство $\Lambda_{0}$ всех зарядов $G$ на $(S, \Im)$, имеющих ограниченную вариацию и таких, что $G(S)=0$. Определим $\tau$-топологию в $\Lambda_{0}$ аналогично ее определению для $\Lambda$. Все топологические свойства в $\Lambda_{0}$ будут рассматриваться относительно $\tau$-топологии.

Для любого $G \in \Lambda_{0}$ и $P \in \Lambda$ определим функционал

$$
\rho_{0}^{2}(G: P)=\int_{S} g^{2} d P, \quad g=\frac{d G}{d P},
$$

если $G$ абсолютно непрерывна относительно $P$, и $\rho_{0}^{2}(G: P)=\infty$ в противном случае. Для любого $\Omega_{0} \subset \Lambda_{0}$ обозначим $\rho_{0}^{2}\left(\Omega_{0}: P\right)=\inf \left\{\rho_{0}^{2}(G: P)\right.$, $\left.G \in \Omega_{0}\right\}$. С точки зрения статистических приложений функционал $\rho_{0}^{2}$ может рассматриваться как естественный аналог информационного количества Фишера.

Сделаем следующее предположение.

А. Существуют открытое множество $\Omega_{0} \subset \Lambda_{0}$ и функция $\omega(t)$, $\omega(t) / t \rightarrow 0$ при $t \rightarrow 0$, такие, что

1) для любой последовательности зарядов $G_{n} \in \Omega_{0}, P+b_{n} G_{n} \in \Lambda$, сушествует последовательность вероятностных мер $Q_{n} \in \Omega_{n}$ такая, что $\rho\left(Q_{n}, P+b_{n} G_{n}\right)<\omega\left(\rho\left(P, P+b_{n} G_{n}\right)\right)$

2) для любой последовательности вероятностных мер $Q_{n} \in \Omega_{n}$ существует последовательность зарядов $G_{n} \in \Omega_{0}, P+b_{n} G_{n} \in \Lambda$, такая, что $\rho\left(Q_{n}, P+b_{n} G_{n}\right)<\omega\left(\rho\left(Q_{n}, P\right)\right)$.

Таким образом, множества $P+b_{n} \Omega_{0}$ допускают интерпретацию как «линейные аппроксимации» множеств $\Omega_{n}$ в метрике Хеллингера. Легко видеть, что $\rho\left(\Omega_{n}, P\right)=\frac{1}{2} b_{n} \rho_{0}\left(\Omega_{0}: P\right)(1+o(1))$ при $n \rightarrow \infty$.

Теорема 3.1 , приведенная ниже, является некоторым вариантом теоремы 3.2 [3] и теоремы 3.1 [8].

Теорема 3.1. Пусть выполнено условие А и пусть $\rho_{0}\left(\operatorname{int}\left(\Omega_{0}\right): P\right)=$ $\rho_{0}\left(\operatorname{cl}\left(\Omega_{0}\right): P\right)$. Тогда

$$
\lim _{n \rightarrow \infty}\left(2 n \rho^{2}\left(\Omega_{n}, P\right)\right)^{-1} \ln P\left(\widehat{P}_{n} \in \Omega_{n}\right)=-1 .
$$

Найдутся заряд $G \in \operatorname{cl}\left(\Omega_{0}\right)$ и последовательность $G_{n} \in \Omega_{0}$, сходящаяся $\kappa G$ в $\tau$-топологии, такие, что $P+b_{n} G_{n} \in \Lambda$ u nри $n \rightarrow \infty$

$$
\begin{aligned}
\rho\left(\Omega_{n}, P\right) & =\rho\left(P+b_{n} G_{n}, P\right)(1+o(1)) \\
& =\frac{1}{2} b_{n} \rho_{0}\left(\Omega_{0}: P\right)(1+o(1))=\frac{1}{2} b_{n} \rho_{0}(G: P)(1+o(1)) .
\end{aligned}
$$

Определим процедуру существенной выборки аналогично п. 2, заменив в этом определении (см. (2.4)) число $b$ на последовательность $b_{n}$, вероятностные меры $Q_{1}, \ldots, Q_{l}$ на последовательности вероятностных мер $Q_{n 1}, \ldots, Q_{n l}$ и вероятностную меру $Q$ на $Q_{n}=p_{1} Q_{n 1}+\cdots+p_{l} Q_{n l}$, а также в $(2.10)$ множество $\Omega$ на последовательность множеств $\Omega_{n}$.

Приводимая ниже лемма 3.1 является аналогом леммы 2.1 . 
Лемма 3.1. Пусть выполнено условие А и пусть $\rho_{0}\left(\Omega_{0}: P\right)=$ $\rho_{0}\left(\operatorname{cl}\left(\Omega_{0}\right): P\right)$. Тогда для любой последовательности прочедур существенной выборки

$$
\liminf _{n \rightarrow \infty}\left(2 n \rho^{2}\left(\Omega_{n}, P\right)\right)^{-1} \ln E \widehat{U}_{n} \geqslant-2,
$$

или, в других терминах,

$$
\liminf _{n \rightarrow \infty}\left(n b_{n}^{2}\right)^{-1} \ln E \widehat{U}_{n} \geqslant-\rho_{0}^{2}\left(\Omega_{0}: P\right) .
$$

Лемма 3.1 непосредственно следует из (2.6) и теоремы 3.1 .

Мы будем говорить, что последовательности вероятностных мер $Q_{n 1}, \ldots, Q_{n l}$ и неотрицательные числа $p_{1}, \ldots, p_{l} \in \mathbf{R}^{1}, p_{1}+\cdots+p_{l}=1$, порождают последовательность асимптотически эффективных процедур существенной выборки, если в (3.3) (соответственно (3.4)) достигается равенство.

Сделаем дополнительные предположения.

C1. Существует только конечное число зарядов $G_{1}, \ldots, G_{m} \in \operatorname{cl}\left(\Omega_{0}\right)$ таких, что $\rho_{0}\left(G_{j}: P\right)=\rho_{0}\left(\Omega_{0}: P\right), 1 \leqslant j \leqslant m$.

Обозначим $g_{j}=d G_{j} / d P, 1 \leqslant j \leqslant m$. Определим множества $\Psi_{j}=$ $\Psi_{G_{j}}=\left\{G: \rho_{0}\left(G+G_{j}: P\right)<2 \rho_{0}\left(\Omega_{0}: P\right), G \in \Lambda_{0}\right\}$ для всех $1 \leqslant j \leqslant m$. Множество $\Psi_{j}$ содержит все заряды $G$, имеюшие плотности $g=\frac{d G}{d P}$, из шара в $L_{2}(P)$ с центром $-g_{j}$ и радиусом $2 \rho_{0}\left(\Omega_{0}: P\right)$. Положим $\Psi=\bigcap_{j=1}^{m} \Psi_{j}$.

C2. $\Omega_{0} \cap \Psi=\varnothing$.

Для каждого $j, 1 \leqslant j \leqslant m$, определим множество $\bar{\Gamma}_{G_{j}}=$ $\left\{H: \int_{S} g_{j} d H<\rho_{0}^{2}\left(\Omega_{0}: P\right), H \in \Lambda_{0}\right\}$. Ясно, что $\bar{\Gamma}_{G_{j}} \supset \Psi_{j}$. Таким образом, С2 может быть заменено более сильным предположением.

C3. $\Omega_{0} \subset \Lambda_{0} \backslash \operatorname{cl}\left(\bigcap_{j=1}^{m} \bar{\Gamma}_{G_{j}}\right)$.

Предположение типа С 3 было сделано в теореме 2.3 и является стандартным при моделировании больших уклонений (см. [14]). Это предположение обычно не выполнено для множеств $\Omega_{0}$, порожденных нелинейными статистическими функционалами, в частности, тестовыми статистиками критерия омега-квадрат. Приведенное выше условие С2 до некоторой степени позволяет обойти эту трудность. Ясно, что С3 влечет $\mathrm{C} 2$.

Теорема 3.2. Пусть выполнены условия А, С1 и С2 или А, С1 и С3. Пусть $\rho_{0}\left(\Omega_{0}: P\right)=\rho_{0}\left(\operatorname{cl}\left(\Omega_{0}\right): P\right)$. Тогда последовательность вероятностных мер $Q_{n j}, 1 \leqslant j \leqslant l$, имеюших плотности

$$
q_{n j}=\lambda_{n j}+b_{n} h_{j} \chi\left(\left|h_{j}\right|<c_{n} b_{n}^{-1}\right),
$$

где $c_{n} b_{n}^{-1} \rightarrow \infty, c_{n} \rightarrow 0 u \lambda_{n j} \rightarrow 1$ nрu $n \rightarrow \infty$, порождает асимптотически эффективнье прочедуры существенной выборки $\widehat{V}_{n t}$ (см. (2.4), (2.10)), если и только если множество $h_{j}, 1 \leqslant j \leqslant l$, содержит множество всех плотностей $g_{i}, 1 \leqslant i \leqslant m, u p_{j} \neq 0$ для всех $j$ таких, что $h_{j}=g_{i}$. 
То же самое утверждение справедливо и для последовательности вероятностных мер $Q_{n j}^{(1)}$, имеющих плотности

$$
q_{n j}^{(1)}=c\left(b_{n}\right) \exp \left\{b_{n} h_{j}\right\} \chi\left(\left|h_{j}\right|<c_{n} b_{n}^{-1}\right),
$$

где $c_{n} b_{n}^{-1} \rightarrow \infty, c_{n} \rightarrow 0$ при $n \rightarrow \infty$. Здесь $c\left(b_{n}\right)$ является нормируюшей константой.

Доказательство теоремы 3.2 будет приведено в п. 4.

3 а м е ч а н и е 3.1. Если функционал $T$ нелинейный, может оказаться, что условие С2 не выполнено. В некоторых случаях тогда может быть применена следующая модификация предложенной процедуры. Предположим, что сушествует конечное число зарядов $G_{m+1}, \ldots, G_{m+a} \in$ $\operatorname{cl}\left(\Omega_{0}\right)$ таких, что $\bigcap_{j=1}^{m+a} \Psi_{G_{j}} \cap \Omega_{0}=\varnothing$. Рассмотрим процедуру существенной выборки, основанную на вероятностных мерах $Q_{n j}$, имеющих плотности $q_{n j}$ или $q_{n j}^{(1)}$, таких, что $h_{j}=g_{j}=\frac{d G_{j}}{d P}, 1 \leqslant j \leqslant m+a$. Тогда анализ доказательства теоремы 3.2 показывает, что подобная модификация процедуры асимптотически эффективна. Таким образом, $u_{n i j}, j>m$, могут интерпретироваться как дополнительные слагаемые регуляризации.

П р и м е р. Пусть $T\left(\widehat{P}_{n}\right)$ является тестовой статистикой критерия типа омега-квадрат и задается функционалом $T(Q)=\int_{0}^{1}(F(x)$ $x)^{2} r(x) d x$, где $F(x)$ обозначает функцию распределения $Q, S=[0,1]$, $r$ - весовая функция, непрерывная на $[0,1]$. Мы предполагаем здесь, что $P$ является мерой равномерного распределения на $[0,1]$.

Множество $\Omega_{0}$ равно

$$
\Omega_{0}=\left\{G: \int_{0}^{1} H^{2}(x) r(x) d x \geqslant 1, H(x)=G((0, x]), G \in \Lambda_{0}\right\} .
$$

Заряды $G$, удовлетворяющие условию $\rho_{0}(G: P)=\rho_{0}\left(\Omega_{0}: P\right), G \in \operatorname{cl}\left(\Omega_{0}\right)$, определяются уравнением (см. [1])

$$
H^{\prime \prime}+\lambda_{1} r H=0, \quad H(0)=H(1)=0,
$$

где $H(x)=G(0, x), x \in(0,1)$, и $\lambda_{1}$ - наибольшее собственное число уравнения (3.7).

Пусть $\lambda_{1}>\lambda_{2}>\cdots$ - собственные числа (3.7), и пусть $k=$ $\max \left\{i: 4 \lambda_{i} \geqslant \lambda_{1}\right\}$. Предположим, что для каждого $\lambda_{j}, 1 \leqslant j \leqslant k$, существует единственная собственная функция $H_{j}$. Тогда заряды $G_{j}$, $G_{j}((0, x))=H_{j}(x)$ и $G_{k+j}((0, x))=-H_{j}(x), 1 \leqslant j \leqslant k$, удовлетворяют условиям теоремы 3.2. Заряды $G_{2}, \ldots, G_{k}, G_{k+2}, \ldots, G_{2 k}$ при этом играют ту же самую роль, что и в замечании 3.1 .

4. Доказательство теоремы 3.2. Рассуждения основаны на стандартной технике доказательства теорем о больших уклонениях эмпирических мер (см. [9]) и ее модификации на случай вероятностей умеренных уклонений (см. [7], [8]). Естественно, методы, развитые для до- 
казательства асимптотической эффективности процедур существенной выборки (см. [14], [5]), также играют значительную роль.

Начнем с доказательства достаточности. Положим $q_{n j}=\lambda_{n j}+$ $b_{n} g_{j} \chi\left(g_{j}>-c_{n} b_{n}^{-1}\right), 1 \leqslant j \leqslant l=m$. Обозначим $\Pi=\Pi_{k}=\left\{S_{i}\right\}_{1}^{k}$ разбиение $S$, состоящее из конечного числа борелевских множеств $S_{i}, 1 \leqslant i \leqslant k$. Скажем, что разбиение $\Pi$ лучше, чем разбиение $\bar{\Pi}(\Pi \geqslant \bar{\Pi})$, если для каждого $A \in \Pi$ найдется множество $B \in \bar{\Pi}$ такое, что $A \subseteq B$.

Для любых $Q \in \Lambda, G \in \Lambda_{0}$ и разбиения $\Pi=\left\{S_{i}\right\}_{1}^{k}$ множества $S$ обозначим

$$
\rho^{2}(Q, P \mid \Pi)=\sum_{i=1}^{k}\left(P^{1 / 2}\left(S_{i}\right)-Q^{1 / 2}\left(S_{i}\right)\right)^{2}, \quad \rho_{0}^{2}(G: P \mid \Pi)=\sum_{i=1}^{k} \frac{G^{2}\left(S_{i}\right)}{P\left(S_{i}\right)} .
$$

Здесь и в дальнейшем мы предполагаем, что $P\left(S_{i}\right) \neq 0$ для всех $1 \leqslant i \leqslant k$.

Известно, что (см. [8])

$$
\rho(Q, P)=\sup \rho(Q, P \mid \Pi), \quad \rho_{0}(G: P)=\sup \rho_{0}(G: P \mid \Pi),
$$

где супремум берется по всем разбиениям П множества $S$.

Проводя рассуждения, аналогичные доказательству леммы 2.4 в [9], нетрудно показать, что

$$
\begin{aligned}
& \rho^{2}\left(\operatorname{cl}\left(\Omega_{n}\right), P\right)=\sup _{\Pi} \rho^{2}\left(\operatorname{cl}\left(\Omega_{n}\right), P \mid \Pi\right), \\
& \rho_{0}^{2}\left(\operatorname{cl}\left(\Omega_{0}\right), P\right)=\sup _{\Pi} \rho_{0}^{2}\left(\operatorname{cl}\left(\Omega_{0}\right), P \mid \Pi\right),
\end{aligned}
$$

где супремум также берется по всем разбиениям П.

Для любых $\delta>0, \delta_{1}>0$ найдутся $C_{1}=C_{1}\left(\delta_{1}\right)>1$ и разбиение $\Pi_{C_{1}, \delta, \delta_{1}}=\left\{W_{i}\right\}_{1}^{k}$ такие, что

$$
\inf _{y \in W_{k}} \max _{1 \leqslant j \leqslant m}\left|g_{j}(y)\right|>C_{1}, \quad \max _{1 \leqslant j \leqslant m} \int_{W_{k}} g_{j}^{2} d P<\delta_{1},
$$

и для любых $1 \leqslant j \leqslant m, 1 \leqslant i \leqslant k-1$ для всех $y \in W_{i}$ имеет место

$$
\max _{x \in W_{i}} g_{j}(x) \leqslant g_{j}(y) \leqslant \min _{x \in W_{i}} g_{j}(x)+\delta .
$$

Обозначим $\Xi=\left\{\Pi: \Pi \geqslant \Pi_{C_{1}, \delta, \delta_{1}}\right\}$. В дальнейшем мы будем рассматривать только разбиения $\Pi$, принадлежащие $\Xi$.

Пусть $\Pi=\left\{S_{i}\right\}_{i=1}^{k} \in \Xi$. Для всех $i, 1 \leqslant i \leqslant k$, и всех $j, 1 \leqslant j \leqslant m$, обозначим $q_{n j i}=Q_{n j}\left(S_{i}\right), g_{j i}=E\left[g_{j}\left(X_{1}\right) \chi\left(X_{1} \in S_{i}, g_{j}\left(X_{1}\right)>-c_{n} b_{n}^{-1}\right)\right]$. Ясно, что $\lambda_{n j}=1-b_{n} \int_{S} g_{j} \chi\left(g_{j}>-c_{n} b_{n}^{-1}\right) d P=1+b_{n} \xi_{n j}=1+o\left(b_{n}\right)$ при $n \rightarrow \infty$.

Отсюда следует, что

$$
q_{n j i}=\lambda_{n j} p_{i}+b_{n} g_{j i}+o\left(b_{n}\right)=p_{i}+b_{n} g_{j i}+o\left(b_{n}\right) .
$$

Рассмотрим сначала случай $m=1$ и $P+b_{n} G_{1} \in \Lambda$, если $n>n_{0}$. Случай произвольного $m$ будет позже сведен к данной постановке. Для всех $i$, 
$1 \leqslant i \leqslant k$, обозначим $r_{i}=\int_{S_{i}} g_{1}^{2} d P, d_{n i}=\int_{S_{i}} q_{n 1}^{-1} d P$. Положим $\gamma_{n}^{2}=$ $\frac{1}{4} b_{n}^{2} \sum_{i=1}^{k} g_{1 i}^{2} / p_{i}$.

Применяя формулу Тейлора, получаем для $1 \leqslant i \leqslant k$

$$
d_{n i}=\left(2-\lambda_{n 1}\right) p_{i}-b_{n} g_{1 i}+b_{n}^{2} r_{i}(1+o(1))=p_{i}-b_{n} g_{1 i}+b_{n}^{2} r_{i}+o\left(b_{n}^{2}\right) .
$$

Имеем

$$
\begin{aligned}
E_{Q_{n}} \widehat{U}_{n} & =E\left[w_{n 1}^{-1} \chi\left(\widehat{P}_{n} \in \Omega_{n}\right)\right] \\
& \leqslant E\left[w_{n 1}^{-1} \chi\left(\rho^{2}\left(\widehat{P}_{n}+b_{n} G_{1}, P \mid \Pi\right)>\rho^{2}\left(\Omega_{n}+b_{n} G_{1}, P \mid \Pi\right)\right)\right] \\
& \leqslant E\left[w_{n 1}^{-1} \chi\left(\rho^{2}\left(\widehat{P}_{n}+b_{n} G_{1}, P \mid \Pi\right)\right]>4 \beta \gamma_{n}^{2}\right) \doteq \bar{I}_{n}(\Pi),
\end{aligned}
$$

где $\beta=\beta(\delta), \beta(\delta) \rightarrow 1$ при $\delta \rightarrow 0$. Заметим, что правая часть (4.6) зависит только от значений $p_{i}, g_{1 i}, d_{n i}, 1 \leqslant i \leqslant k$, и $\beta \gamma_{n}^{2}$.

Применяя формулу Стирлинга, получаем

$$
\begin{aligned}
\bar{I}_{n}(\Pi)<\sum^{\prime} \frac{n !}{\left(n z_{n 1}\right) ! \cdots\left(n z_{n k}\right) !} \prod_{i=1}^{k} d_{n i}^{n z_{n i}} \\
\leqslant C(2 \pi)^{-k / 2} \sum^{\prime} \exp \left\{\left(\frac{1}{2}-\frac{k}{2}\right) \ln n-\frac{1}{2} \sum_{i=1}^{k} \ln z_{n i}\right. \\
\left.\quad-n \sum_{i=1}^{k} z_{n i} \ln \frac{z_{n i}}{p_{i}}+n \sum_{i=1}^{k} z_{n i} \ln \frac{d_{n i}}{p_{i}}\right\} \doteq I\left(Z_{n}^{(1)}\right) .
\end{aligned}
$$

Здесь суммирование $\sum^{\prime}$ проводится по множеству $Z_{n}^{(1)}$ всех $z=$ $\left(z_{n 1}, \ldots, z_{n k}\right)$ таких, что $n z_{n 1}, \ldots, n z_{n k}$ - неотрицательные целые числа, $z_{n 1}+\cdots+z_{n k}=1$ и

$$
J_{n 1}(z)=\sum_{i=1}^{k}\left(\left(z_{n i}+b_{n} g_{1 i}\right)^{1 / 2}-p_{i}^{1 / 2}\right)^{2}>4 \beta \gamma_{n}^{2}\left(1+b_{n}^{1 / 2}\right) .
$$

Определим также множество $Z_{n}^{(2)}$ всех $z=\left(z_{n 1}, \ldots, z_{n k}\right)$ таких, что $n z_{n 1}, \ldots, n z_{n k}$ - неотрицательные целые числа, $z_{n 1}+\cdots+z_{n k}=1$ и

$$
J_{n 2}(z)=\sum_{i=1}^{k}\left(z_{n i}^{1 / 2}-p_{i}^{1 / 2}\right)^{2}>\beta \gamma_{n}^{2} .
$$

Ясно, что $Z_{n}^{(1)} \subset Z_{n}^{(2)}$ для всех $n>n_{0}\left(\delta, C_{1}\right)$ и, следовательно, $I\left(Z_{n}^{(1)}\right) \leqslant$ $I\left(Z_{n}^{(2)}\right)$.

Зафиксируем $\varepsilon>0$ и для $s=0,1,2, \ldots$ определим множества $Z_{n s}^{(2)}=\left\{z:(1+\varepsilon s) \gamma_{n}^{2}<J_{n 2}(z) \leqslant(1+\varepsilon(s+1)) \gamma_{n}^{2}, z \in Z_{n}^{(2)}\right\}$. Асимптотика числа элементов $Z_{n s}^{(2)}$ совпадает с аналогичной асимптотикой для $U_{n s}^{(2)}=\left\{u: 4(1+\varepsilon s) \gamma_{n}^{2} \leqslant \sum_{i=1}^{k} u_{i}^{2} / p_{i} \leqslant 4(1+\varepsilon(s+1)) \gamma_{n}^{2}, u=\left(u_{1}, \ldots, u_{k}\right)\right.$, $n u_{1}, \ldots, n u_{k}$ - целые числа, $\left.u_{1}+\cdots+u_{k}=0\right\}$ и равна (см. [8])

$$
C \varepsilon\left(\Gamma\left(\frac{k}{2}\right)\right)^{-1}(2 \pi)^{\zeta-1}\left(n \gamma_{n}\right)^{2 \zeta}(1+\varepsilon s)^{\zeta-1} \prod_{i=1}^{k} p_{i}^{1 / 2}(1+o(1))
$$


при $n \rightarrow \infty$. Здесь $\zeta=(k-1) / 2$ и $\Gamma(k / 2)-$ значение гамма-функции для $k / 2$.

Разлагая $z_{n i} \ln \left(z_{n i} / p_{i}\right)$ и $z_{n i} \ln \left(d_{n i} / p_{i}\right)$ в ряд Тейлора по степеням $\left(z_{n i}^{1 / 2}-p_{i}^{1 / 2}\right) p_{i}^{-1 / 2}$ и используя (4.4), (4.5), для любого $z \in Z_{n s}^{(2)}$ получаем

$$
\begin{aligned}
-\sum_{i=1}^{k} z_{n i} \ln \frac{z_{n i}}{p_{i}}= & -2 \sum_{i=1}^{k}\left(z_{n i}^{1 / 2}-p_{i}^{1 / 2}\right)^{2}(1+o(1)) \\
\sum_{i=1}^{k} z_{n i} \ln \frac{d_{n i}}{p_{i}}= & \sum_{i=1}^{k} p_{i}\left(1+2\left(z_{n i}^{1 / 2}-p_{i}^{1 / 2}\right) p_{i}^{-1 / 2}+\left(z_{n i}^{1 / 2}-p_{i}^{1 / 2}\right)^{2} p_{i}^{-1}\right) \\
& \times \ln \left(1-\left(\lambda_{n 1}-1\right)-b_{n} \frac{g_{1 i}}{p_{i}}+b_{n}^{2} \frac{r_{i}}{p_{i}}(1+o(1))\right) \\
= & -2 b_{n} \sum_{i=1}^{k} g_{1 i}\left(z_{n i}^{1 / 2}-p_{i}^{1 / 2}\right) p_{i}^{-1 / 2} \\
& -\frac{1}{2} b_{n}^{2} \sum_{i=1}^{k} \frac{g_{1 i}^{2}}{p_{i}}+b_{n}^{2} \sum_{i=1}^{k} r_{i}+o\left(b_{n}^{2}\right) .
\end{aligned}
$$

При доказательстве последнего равенства в (4.12) мы использовали соотношение $\lambda_{n 1}=1-b_{n} \sum_{i=1}^{k} g_{1 i}$.

Применяя (4.4), получаем

$$
-2 b_{n} \sum_{i=1}^{k} g_{1 i}\left(z_{n i}^{1 / 2}-p_{i}^{1 / 2}\right) p_{i}^{-1 / 2}=-4 \sum_{i=1}^{k}\left(z_{n i}^{1 / 2}-p_{i}^{1 / 2}\right)\left(q_{n 1 i}^{1 / 2}-p_{i}^{1 / 2}\right)(1+o(1)) \text {. }
$$

Так как $\Pi \in \Xi$ имеем

$$
\begin{gathered}
-\frac{1}{2} b_{n}^{2} \sum_{i=1}^{k} \frac{g_{1 i}^{2}}{p_{i}}+b_{n}^{2} \sum_{i=1}^{k} r_{i} \leqslant \frac{1}{2} b_{n}^{2} \sum_{i=1}^{k} \frac{g_{1 i}^{2}}{p_{i}}+C C_{1} \delta b_{n}^{2}+C \delta_{1} b_{n}^{2} \\
\leqslant 2 \sum_{i=1}^{k}\left(q_{n 1 i}^{1 / 2}-p_{i}^{1 / 2}\right)^{2}+O\left(C_{1} \delta b_{n}^{2}+\delta_{1} b_{n}^{2}\right)+o\left(b_{n}^{2}\right) .
\end{gathered}
$$

Из (4.11)-(4.14) получаем

$$
\begin{aligned}
- & \sum_{i=1}^{k} z_{n i} \ln \frac{z_{n i}}{p_{i}}+\sum_{i=1}^{k} z_{n i} \ln \frac{d_{n i}}{p_{i}} \\
= & 4 \sum_{i=1}^{k}\left(z_{n i}^{1 / 2}-p_{i}^{1 / 2}\right)^{2}+2 \sum_{i=1}^{k}\left(z_{n i}^{1 / 2}-q_{n 1 i}^{1 / 2}\right)^{2}+O\left(\delta_{1} b_{n}^{2}+C_{1} \delta b_{n}^{2}\right)+o\left(b_{n}^{2}\right) \\
= & -2\left(z_{n i}+2 z_{n i}^{1 / 2} q_{n 1 i}^{1 / 2}+q_{n 1 i}-4 z_{n i}^{1 / 2} p_{i}^{1 / 2}+4 p_{i}-4 q_{n 1 i}^{1 / 2} p_{i}^{1 / 2}\right) \\
& +4\left(q_{n i}-2 q_{n 1 i}^{1 / 2} p_{i}^{1 / 2}+p_{i}\right)+O\left(\delta_{1} b_{n}^{2}+C_{1} \delta b_{n}^{2}\right)+o\left(b_{n}^{2}\right) \\
= & -2 \sum_{i=1}^{k}\left(z_{n i}^{1 / 2}+q_{n 1 i}^{1 / 2}-2 p_{i}^{1 / 2}\right)^{2} \\
& +4 \sum_{i=1}^{k}\left(q_{n 1 i}^{1 / 2}-p_{i}^{1 / 2}\right)^{2}+O\left(\delta_{1} b_{n}^{2}+C_{1} \delta b_{n}^{2}\right)+o\left(b_{n}^{2}\right)
\end{aligned}
$$


Применяя (4.4), (4.5), непосредственными вычислениями получаем

$$
\begin{aligned}
& \sum_{i=1}^{k}\left(z_{n i}^{1 / 2}+q_{n 1 i}^{1 / 2}-2 p_{i}^{1 / 2}\right)^{2}=\sum_{i=1}^{k}\left(z_{n i}^{1 / 2}-\left(p_{i}-b_{n} g_{1 i}\right)^{1 / 2}\right)(1+o(1)) \\
& \geqslant \sum_{i=1}^{k}\left(\left(z_{n i}+b_{n} g_{1 i}\right)^{1 / 2}-p_{i}^{1 / 2}\right)^{2}(1+o(1)) \\
& \geqslant(4+2 \varepsilon s) \gamma_{n}^{2}+o\left(b_{n}^{2}\right)=(4+2 \varepsilon s) \sum_{i=1}^{k}\left(q_{n 1 i}^{1 / 2}-p_{i}^{1 / 2}\right)^{2}+o\left(b_{n}^{2}\right)
\end{aligned}
$$

если $z \in Z_{n s}^{(2)} \cap Z_{n}^{(1)}$ и $J_{n 1}(z)>\beta \gamma_{n}^{2}\left((1+\varepsilon s)^{1 / 2}+1\right)^{2}$. Доказательство первого неравенства в $(4.16)$ основано на соотношении $z_{n i}^{1 / 2}-\left(p_{i}-b_{n} g_{1 i}\right)^{1 / 2}=$ $\left(\left(z_{n i}+b_{n} g_{1 i}\right)^{1 / 2}-p_{i}^{1 / 2}\right)^{2}(1+o(1))$, которое доказывается непосредственными вычислениями.

Из (4.15), (4.16) следует, что

$$
\sum_{i=1}^{k} z_{n i} \ln \frac{z_{n i}}{p_{i}}+\sum_{i=1}^{k} z_{n i} \ln \frac{d_{n i}}{p_{i}} \leqslant-(4+2 \varepsilon s) \gamma_{n}^{2}+O\left(\delta_{1} b_{n}^{2}+C_{1} \delta b_{n}^{2}\right)+o\left(b_{n}^{2}\right) .
$$

Отсюда и из (4.6)-(4.10), (4.15), (4.16), С2 вытекает, что

$$
\begin{aligned}
I\left(Z_{n}^{(1)}\right) \leqslant & \sum_{s=0}^{\infty} I\left(Z_{n s}^{(2)} \cap Z_{n}^{(1)}\right) \leqslant C \sum_{s=0}^{\infty}(1+\varepsilon s)^{\zeta-1} \varepsilon\left(n \gamma_{n}^{2}\right)^{\zeta} \\
& \times \exp \left\{-n(4+2 \varepsilon s) \gamma_{n}^{2}(1+o(1))+O\left(C_{1} \delta b_{n}^{2}+\delta_{1} b_{n}^{2}\right)\right\} .
\end{aligned}
$$

Выберем последовательность $\varepsilon=\varepsilon_{n}=o(1)$ такую, что $n \varepsilon_{n} \gamma_{n}^{2} \rightarrow \infty$ при $n \rightarrow \infty$. Тогда из (4.18) следует, что

$$
I\left(Z_{n}^{(1)}\right)<\exp \left\{-4 n \gamma_{n}^{2}(1+o(1))+O\left(\left(C_{1} \delta+\delta_{1}\right) b_{n}^{2}\right)\right\}
$$

при $n \rightarrow \infty$.

Из (4.19) и (4.3) при надлежащем стремлении $\delta \rightarrow 0, \delta_{1} \rightarrow 0$ таком, что $C_{1}\left(\delta_{1}\right) \delta \rightarrow 0$, получаем, что

$$
E_{Q_{n}} \widehat{U}_{n}<\exp \left\{-4 n \rho^{2}\left(\Omega_{n}, P\right)(1+o(1))\right\}
$$

при $n \rightarrow \infty$.

Предположим, что $m$ произвольно. Тогда

$$
\begin{aligned}
E_{Q_{n}} \widehat{U}_{n} & =E\left\{\left(\sum_{j=1}^{l} p_{j} u_{n j}\right)^{-1} \chi\left(\widehat{P}_{n} \in \Omega_{n}\right)\right\} \\
\leqslant & E\left\{( \sum _ { j = 1 } ^ { l } p _ { j } u _ { n j } ) ^ { - 1 } \chi \left(\max _{1 \leqslant t \leqslant l} \rho\left(\widehat{P}_{n}+b_{n} G_{n t}, P \mid \Pi\right)\right.\right. \\
& \left.\left.\geqslant \frac{1}{2} \beta b_{n} \max _{1 \leqslant t \leqslant l} \rho_{0}\left(\left(\Omega_{0}+G_{t}\right): P \mid \Pi\right)\right)\right\}
\end{aligned}
$$




$$
\begin{array}{r}
\leqslant \sum_{t=1}^{l} E\left\{( \sum _ { j = 1 } ^ { l } p _ { j } u _ { n j } ) ^ { - 1 } \chi \left(\rho\left(\widehat{P}_{n}+b_{n} G_{n t}, P \mid \Pi\right)\right.\right. \\
\left.\left.\geqslant \frac{1}{2} \beta b_{n} \rho_{0}\left(\left(\Omega_{0}+G_{t}\right): P \mid \Pi\right)\right)\right\} \\
\leqslant \sum_{t=1}^{l} p_{t}^{-1} E\left\{\left(u _ { n t } ^ { - 1 } \chi \left(\rho\left(\widehat{P}_{n}+b_{n} G_{n t}, P \mid \Pi\right)\right.\right.\right. \\
\left.\left.\geqslant \frac{1}{2} \beta b_{n} \rho_{0}\left(\left(\Omega_{0}+G_{t}\right): P \mid \Pi\right)\right)\right\} .
\end{array}
$$

Таким образом, задача свелась к рассмотренному выше случаю $m=1$.

При доказательстве теоремы в части необходимости проведем рассуждения, аналогичные рассуждениям в [14]. Предположим, что $p_{1}=0$ (если $p_{j}=0$ для некоторого $j, 2 \leqslant j \leqslant m$, рассуждения аналогичны). Тогда существуют последовательности $\kappa_{l}>\kappa_{1 l}>0, \kappa_{l} \rightarrow 0$ при $l \rightarrow \infty$, и разбиения $\Pi(l) \in \Xi$ такие, что

$$
\Delta_{l}=\left\{G: \rho_{0}\left(G-\left(1+\kappa_{l}\right) G_{1}: P \mid \Pi_{l}\right)<\kappa_{l} \gamma_{n}, \quad G \in \Lambda_{0 \Phi}\right\} \subset \Omega_{0} .
$$

Здесь и в дальнейшем $k, \gamma_{n}, q_{n j i}, g_{1 i}, 1 \leqslant j \leqslant m, 1 \leqslant i \leqslant k$, определяются по разбиению $\Pi_{l}$ аналогично доказательству достаточности. Таким образом, получаем

$$
\begin{aligned}
E_{Q_{n}} \widehat{U}_{n} & \geqslant E\left[\left(\sum_{j=2}^{k} p_{j} u_{n j}\right)^{-1} \chi\left(\widehat{P}_{n} \in P+b_{n} \Delta_{l}\right)\right] \\
& \geqslant k^{-1} \min _{2 \leqslant j \leqslant m} E\left[u_{n j}^{-1} \chi\left(\widehat{P}_{n} \in P+b_{n} \Delta_{l}\right)\right] \geqslant C I\left(D_{n}\right),
\end{aligned}
$$

где $I\left(D_{n}\right)$ задается так же, как в (4.7), а $D_{n}-$ множество всех $z=$ $\left(z_{n 1}, \ldots, z_{n k}\right)$ таких, что $n z_{n 1}, \ldots, n z_{n k}$ - неотрицательные целые числа, $z_{n 1}+\cdots+z_{n m}=1$ и

$$
J_{n 3}(z)=\sum_{i=1}^{k}\left(z_{n i}^{1 / 2}-\left(p_{i}+\left(1+\kappa_{l}\right) b_{n} g_{1 i}\right)^{1 / 2}\right)^{2}<\kappa_{l}^{2} \gamma_{n}^{2}
$$

Тогда число элементов $D_{n}$ не превосходит $C\left(n \gamma_{n} \kappa_{1 l}\right)^{2 \zeta} \prod_{i=1}^{k} p_{i}^{1 / 2}$. Отсюда, рассуждая аналогично (4.6)-(4.10), (4.15), (4.16), получаем

$$
\begin{aligned}
I\left(D_{n}\right) \geqslant & \min _{2 \leqslant j \leqslant m} \exp \left\{-2 n \sum_{i=1}^{k}\left(\left(p_{i}+\left(1+\kappa_{l}\right) b_{n} g_{1 i}\right)^{1 / 2}+q_{n j i}^{1 / 2}-2 p_{i}^{1 / 2}\right)^{2}\right. \\
& \left.+4 n \sum_{i=1}^{k}\left(q_{n j i}^{1 / 2}-p_{i}^{1 / 2}\right)^{2}+O\left(n\left(C_{1} \delta+\delta_{1}\right) b_{n}^{2}\right)+o\left(n b_{n}^{2}\right)\right\} .
\end{aligned}
$$

Так как неравенство (4.24) имеет место для любого $l$ и любого разбиения $\Pi>\Pi_{l}$, после соответствующего предельного перехода $l \rightarrow \infty$, 


$$
\begin{gathered}
\delta=\delta(n) \rightarrow 0, \delta_{1}=\delta_{1}(n) \rightarrow 0, C_{1}=C_{1}(n, \delta) \rightarrow \infty \text { при } n \rightarrow \infty, \text { получаем } \\
E_{Q_{n}} \widehat{U}_{n}>\min _{2 \leqslant j \leqslant m} \exp \left\{-\frac{1}{2} n b_{n}^{2} \int_{S}\left(g_{1}+g_{j}\right)^{2} d P(1+o(1))\right. \\
\left.\quad+n b_{n}^{2} \int_{S} g_{j}^{2} d P+o\left(n b_{n}^{2}\right)\right\} .
\end{gathered}
$$

Таким образом, $E_{Q_{n}} \widehat{U}_{n}$ имеет больший порядок, чем $I\left(Z_{n}^{(1)}\right)$, и мы приходим к противоречию. Доказательство теоремы 3.2 закончено.

\section{СПИСОК ЛИТЕРАТУРЫ}

1. Anderson T. W., Darling D. A. Asymptotic theory of certain «goodness of fit» criteria based on stochastic processes. - Ann. Math. Statist., 1952, v. 23, p. 193-212.

2. Barone P., Gigli A., Piccioni M. Optimal importance sampling for some quadratic forms of ARMA process. - IEEE Trans. Inform. Theory, 1995, v. 45, №6, part 2, p. $1834-1844$.

3. Боровков $A$. A., Могульский $A$. A. О вероятностях больших уклонений в топологических пространствах. II. - Сиб. матем. журн., 1980, т. 21, № 5, с. 12-26.

4. Bucklew J. A. Large Deviations Techniques in Decision, Simulation and Estimation. New York: Wiley, 1990, 270 p.

5. Bucklew J. A, Ney P., Sadowsky J.S. Monte Carlo simulation and large deviation theory for uniformly recurrent Markov chains. - J. Appl. Probab., 1990, v. 27, № 1, p. 44-59.

6. Chen J.-C., Lu D., Sadowsky J.S., Yao K. On importance sampling in digital communications. I: Fundamentals. II: Trellis coded modulation. - IEEE J. Selected Areas Commun., 1993, v. 11, № 3, p. 289-299; 300-308.

7. Ермаков М. С. Большие уклонения эмпирических мер и проверка гипотез. Записки научн. семин. ЛОМИ, 1993, т. 207, с. 37-59.

8. Ермаков М. С. Асимптотическая минимаксность критериев типа Колмогорова и омега-квадрат. - Теория вероятн. и ее примен., 1995 , т. 40 , в. 1 , с. $54-67$.

9. Groeneboom P., Oosterhoff J., Ruymgaart F. H. Large deviation theorems for empirical probability measures. - Ann. Probab., 1979, v. 7, № 4, p. 553-586.

10. Hall P. The Bootstrap and Edgeworth Expansions. New York: Springer-Verlag, 1992, $352 \mathrm{p}$.

11. Johns M. Importance sampling for bootstrap confidence intervals. - J. Amer. Statist. Assoc., 1988, v. 83, № 403, p. 709-714.

12. Lehtonen T., Nyrhinen $H$. On asymptotically efficient simulation of ruin probabilities in a Markovian environment. - Scand. Actuar. J., 1992, № 1, p. 60-75.

13. Sadowsky J.S. On Monte Carlo estimation of large deviations probabilities. - Ann. Appl. Probab., 1996, v. 6, № 2, p. 399-422.

14. Sadowsky J.S., Bucklew J. A. On large deviation theory and asymptotically efficient Monte Carlo estimation. - IEEE Trans. Inform. Theory, 1990, v. 36, № 3, p. 579-588.

15. Siegmund $D$. Importance sampling in the Monte Carlo study of sequential tests. Ann. Statist., 1976, v. 4, p. 673-684.

16. Srinivasan R. Importance Sampling. Applications in Communications and Detection. Berlin: Springer-Verlag, 2002. 